\title{
INMUNOENSAYO MULTIPLEX PARA EL DIAGNÓSTICO SIMULTÁNEO DE DIABETES MELLITUS AUTOINMUNE Y ENFERMEDAD CELÍACA
}

\author{
MULTIPLEX IMMUNOASSAY FOR SIMULTANEOUS DIAGNOSTIC \\ OF AUTOIMMUNE DIABETES MELLITUS AND CELIAC DISEASE
}

\author{
Silvina S. Bombicino ${ }^{1,8}$, Adriana V. Sabljic ${ }^{2,8}$, Natalia I. Faccinetti ${ }^{3,8}$, Luciano L. Guerra ${ }^{4,8}$, \\ Juan I. Marfía ${ }^{5,8}$, Ivana Masci ${ }^{5,8}$, Aldana Trabucchi ${ }^{1,8}$, Edgardo Poskus ${ }^{6,8}$, Silvina N. Valdez ${ }^{7,8}$
}

\section{RESUMEN}

Introducción: la diabetes mellitus autoinmune (DMA) y la enfermedad celíaca (EC) son enfermedades crónicas, poligénicas y multifactoriales vinculadas con la disfunción del sistema inmune. Dado que es frecuente que un mismo paciente presente ambas patologías, la detección simultánea de los marcadores de autoinmunidad de DMA y EC sería una estrategia racional para mejorar el diagnóstico.

Objetivos: desarrollar un inmunoensayo basado en citometría de flujo (FloCMIA multiplex) para la detección simultánea y discriminativa de marcadores de DMA (GADA e IA-2A) y de EC (tTgA).

Materiales y métodos: las muestras analizadas consistieron en sueros provenientes de 35 individuos controles normales y 21 pacientes con diabetes mellitus tipo 1 (DM1). Se empleó un modelo de "doble paratope" incubando los sueros con una mezcla de microesferas de diferente fluorescencia interna, cada una adsorbida con un autoantígeno: $\operatorname{Tr} x G A D, \operatorname{Tr} x \mid \mathrm{A}-2 \circ \mathrm{H}_{6}-\mathrm{tTg}$, y una mezcla de dichos autoantígenos biotinilados. Los inmunocomplejos se detectaron con estreptavidina-ficoeritrina y se adquirió en un citómetro de flujo.

Resultados: FloCMIA multiplex detectó GADA en el 76,2\% de los pacientes e IA-2A en el 52,38\% (sensibilidad analítica: 88,24 y $56,25 \%$ respectivamente,y especificidad: $85,71 \%$ ) y tTgA en el 42,86\% (sensibilidad analítica: 50,0\%, y especificidad: $80,0 \%)$. Estos resultados se contrastaron con el ensayo de unión de radioligando para GADA e IA-2A y se detectaron 80,95 y $76,19 \%$ de los sueros respectivamente (especificidad: $100 \%$ ), y con un ELISA para tTgA se detectó un 38,1\% (especificidad: 97,1\%).

Conclusiones: FloCMIA multiplex permitió detectar y discriminar GADA, IA-2A y/o tTgA, -en un único acto analítico- en sueros de pacientes con DMA y/o EC. El novedoso inmunoensayo desarrollado simplifica el screening de la población a gran escala.

Palabras clave: diabetes mellitus autoinmune; enfermedad celíaca; autoanticuerpos; inmunoensayo multiplex.

Revista de la Sociedad Argentina de Diabetes 2020; Vol. 54 (3-14)

\section{ABSTRACT}

Introduction: autoimmune diabetes mellitus (ADM) and celiac disease (CD) are chronic, polygenic and multifactorial diseases associated with immune system dysfunction. As it is frequent that a patient presents both pathologies, the simultaneous detection of autoimmunity markers of ADM and CD would be a rational strategy to improve the diagnosis.

Objectives: to develop an immunoassay based on Flow Cytometry (FloCMIA multiplex) for the simultaneous and discriminative detection of markers for ADM (GADA and IA-2A) and $C D(t \operatorname{Tg} A)$.

Materials and methods: thirty five serum samples of control individuals and 21 type 1 diabetes mellitus (T1DM) patients were assayed. A "double bridge" model was used for the assay, incubating the serum samples with a mixture of microspheres containing different amount of internal fluorescence, each one adsorbed with an autoantigen: TrXGAD, TrxIA-2 or $\mathrm{H}_{6}-\mathrm{tg}$, and a mixture of the same biotinilated autoantigens. The immunocomplexes were detected using streptavidinphycoerytrin and then acquired in a flow cytometer.

Results: FloCMIA multiplex detected GADA in $76.2 \%$ of the patients; IA-2A in 52.38\% (analytical sensitivity: $88.24 \%$ and $56.25 \%$ respectively, and specificity: $85.71 \%$ ) and $\operatorname{tg} A$ in 42.86\% (analytical sensitivity: $50.0 \%$, and specificity: $80.0 \%$ ). These results were compared with the radioligand binding assay for GADA and IA-2A, detecting $80.95 \%$ and $76.19 \%$ of the serum samples respectively (100\% specificity), and with an ELISA for tTgA detecting $38.1 \%$ (97.1\% specificity).

Conclusions: FloCMIA multiplex allowed detecting and discriminating GADA, IA-2A and/or TTgA, -in a single assay- in serum samples of ADM and/or CD. The novel developed immunoassay simplifies the screening of the large scale population.

Key words: autoimmune diabetes mellitus; celiac disease; autoantibodies; multiplex immunoassay.

Revista de la Sociedad Argentina de Diabetes 2020; Vol. 54 (3-14) 
1 Doctora de la Universidad de Buenos Aires (UBA), Investigadora Asistente, Consejo Nacional de Investigaciones Científicas y Técnicas (CONICET), Ciudad Autónoma de Buenos Aires, Argentina

2 Bioquímica, Universidad de Buenos Aires (UBA), Ciudad Autónoma de Buenos Aires, Argentina

3 Doctora de la Universidad de Buenos Aires (UBA), Ciudad Autónoma de Buenos Aires, Argentina

4 Doctor de la Universidad de Buenos Aires (UBA), Ciudad Autónoma de Buenos Aires, Argentina

5 Estudiante de Bioquímica, Universidad de Buenos Aires (UBA), Ciudad Autónoma de Buenos Aires, Argentina

6 Doctor en Bioquímica, Universidad de Buenos Aires (UBA), Profesor Consulto, Facultad de Farmacia y Bioquímica (UBA), Investigador Contratado CONICET, Ciudad Autónoma de Buenos Aires, Argentina

7 Doctora de la Universidad de Buenos Aires (UBA), Profesora Adjunta, Facultad de Farmacia y Bioquímica (UBA),
Investigadora Independiente CONICET, Ciudad Autónoma de Buenos Aires, Argentina

8 Universidad de Buenos Aires (UBA), Facultad de Farmacia y Bioquímica, Cátedra de Inmunología, Consejo Nacional de Investigaciones Científicas y Técnicas (CONICET), Universidad de Buenos Aires, Instituto de Estudios de Inmunidad Humoral Prof. Ricardo A. Margni (IDEHU), Ciudad Autónoma de Buenos Aires, Argentina

Contacto de la autora: Silvina N. Valdez

E-mail: silval@ffyb.uba.ar

Correspondencia: Junín 956, 4 piso (C1113AAD), Ciudad

Autónoma de Buenos Aires, Argentina

Fecha de trabajo recibido: 19/07/19

Fecha de trabajo aceptado: 09/12/19

Conflictos de interés: los autores declaran que no existe conflicto de interés

\section{INTRODUCCIÓN}

La diabetes mellitus autoinmune (DMA) y la enfermedad celíaca (EC) son patologías poligénicas y multifactoriales vinculadas con una disfunción del sistema inmune.

La diabetes mellitus (DM) constituye un grupo heterogéneo de patologías, las cuales conducen a una elevación de la glucosa en sangre. La DM afecta a 189 millones de personas en el mundo y se calcula que en el año 2020 habrá 324 millones de afectados, lo que implica un aumento del $72 \%$ y la convierte en una pandemia. En América Latina viven 10.400 .000 individuos diabéticos y se calcula que en 2020 habrá un aumento del 88\%, es decir, 20 millones de afectados.

Las estadísticas en Argentina muestran que aproximadamente un $7 \%$ de la población tiene diabetes, aunque la prevalencia se eleva a un $20 \%$ en aquellas personas mayores de 60 años. Es decir, en una población de 40 millones hay 2.800.000 enfermos, de los cuales el $90 \%$ padece DM tipo 2 (DM2) y el 10\% restante DM tipo 1 (DM1). Además existe un serio problema en la salud pública nacional dado que se estima que el $50 \%$ de los afectados ignora que padece la enfermedad. Si bien se distinguen dos tipos principales de DM, en algunos casos es difícil diferenciar entre las dos variantes principales de la enfermedad'.

De esta manera se han propuesto nuevas categorías, entre ellas, la diabetes autoinmune latente del adulto (conocida como LADA por su sigla en inglés) para identificar un subgrupo de pacientes adultos que posee autoinmunidad e inicialmente no requiere insulina pero puede requerirla poco tiempo después del diagnóstico². Aunque la
DM1 fue considerada una enfermedad pediátrica, aproximadamente una cuarta parte de las personas afectadas es diagnosticada en la edad adulta. Asimismo la tasa de DM2 se ha intensificado exponencialmente en niños desde principios de 1990 debido a la epidemia mundial de obesidad. El aumento simultáneo en la incidencia de DM1 y DM2 originó un considerable solapamiento en las poblaciones de pacientes y generó un cambio de paradigma para estas enfermedades otrora fácilmente distinguibles. Aunque los síntomas agudos, y a veces letales, de la diabetes pueden controlarse, sus complicaciones tardías exhiben alta morbilidad y reducen significativamente las expectativas de vida.

El diagnóstico preciso de los distintos tipos de DM permite la implementación del tratamiento adecuado en forma oportuna. De este modo es posible retrasar la aparición de las complicaciones crónicas de la enfermedad al impactar fuertemente en los beneficios para el paciente, así como en la reducción de los costos sociales de la DM. La detección de los autoanticuerpos (marcadores) involucrados en la DMA es importante para favorecer el diagnóstico precoz de las distintas formas de diabetes con componente autoinmune. Los principales marcadores de DMA son los autoanticuerpos anti-insulina o proinsulina (IAA/PAA), autoanticuerpos anti-glutamato decarboxilasa (GADA), autoanticuerpos anti-proteína tirosina fosfatasa asociada a insulinoma 2 (IA-2A) y autoanticuerpos anti-transportador de zinc 8 (ZnT8A).

Por otra parte, la EC es una enteropatía desencadenada por la ingesta de gluten en individuos genéticamente susceptibles (alelos HLA-DQ2 y/o 
DQ8), en quienes se produce un síndrome de malabsorción alimentaria que perdura a lo largo de toda su vida. La prevalencia en Europa y América del Norte es de aproximadamente 1:1003,4; sin embargo, la mayoría de los pacientes es subdiagnosticado o diagnosticado con significativo retraso. $\mathrm{Si}$ bien el diagnóstico definitivo de la EC se realiza mediante el estudio histopatológico en biopsias de yeyuno obtenidas antes y después de iniciado el tratamiento con dietas libre de gluten, se trata de un procedimiento quirúrgico que genera un gran estrés en el paciente y no siempre se evidencian alteraciones histopatológicas concluyentes. En este sentido, los test serológicos para la detección de ciertos anticuerpos representan una alternativa útil para el diagnóstico y seguimiento de pacientes celíacos. La indicación de dieta libre de gluten es un tratamiento eficaz, y el diagnóstico precoz mediante la detección de autoanticuerpos anti-transglutaminasa tisular (tTgA) ha sido ampliamente recomendado 5 .

El subdiagnóstico y/o el diagnóstico impreciso de estas patologías representan una pesada carga socioeconómica; además no existen en nuestro país desarrollos locales de kits para el diagnóstico y seguimiento en DMA y EC, por lo cual el sistema de salud depende de la importación de insumos lo que incrementa sustancialmente el costo de las determinaciones.

Dado que la DMA y la EC comparten alelos de susceptibilidad genética HLA de clase II y no HLA, y se presentan en simultaneidad en un $10 \%$ de los pacientes $^{6}$, la Asociación Americana de Diabetes (ADA) recomienda el screening de rutina para la EC en los pacientes con reciente diagnóstico de DM17. De esta manera, el screening combinado para DM1 y EC sería un apareamiento racional interesante para mejorar y optimizar el diagnóstico de estas patologías relacionadas. Asimismo, los ensayos de prevención de DM1 están en marcha y probablemente se expandirán a múltiples intervenciones posibles. Sin embargo, el screening masivo de los sujetos elegibles aún es un cuello de botella laborioso, por lo cual una estrategia de detección simultánea para marcadores de ambas patologías resulta sumamente interesante.

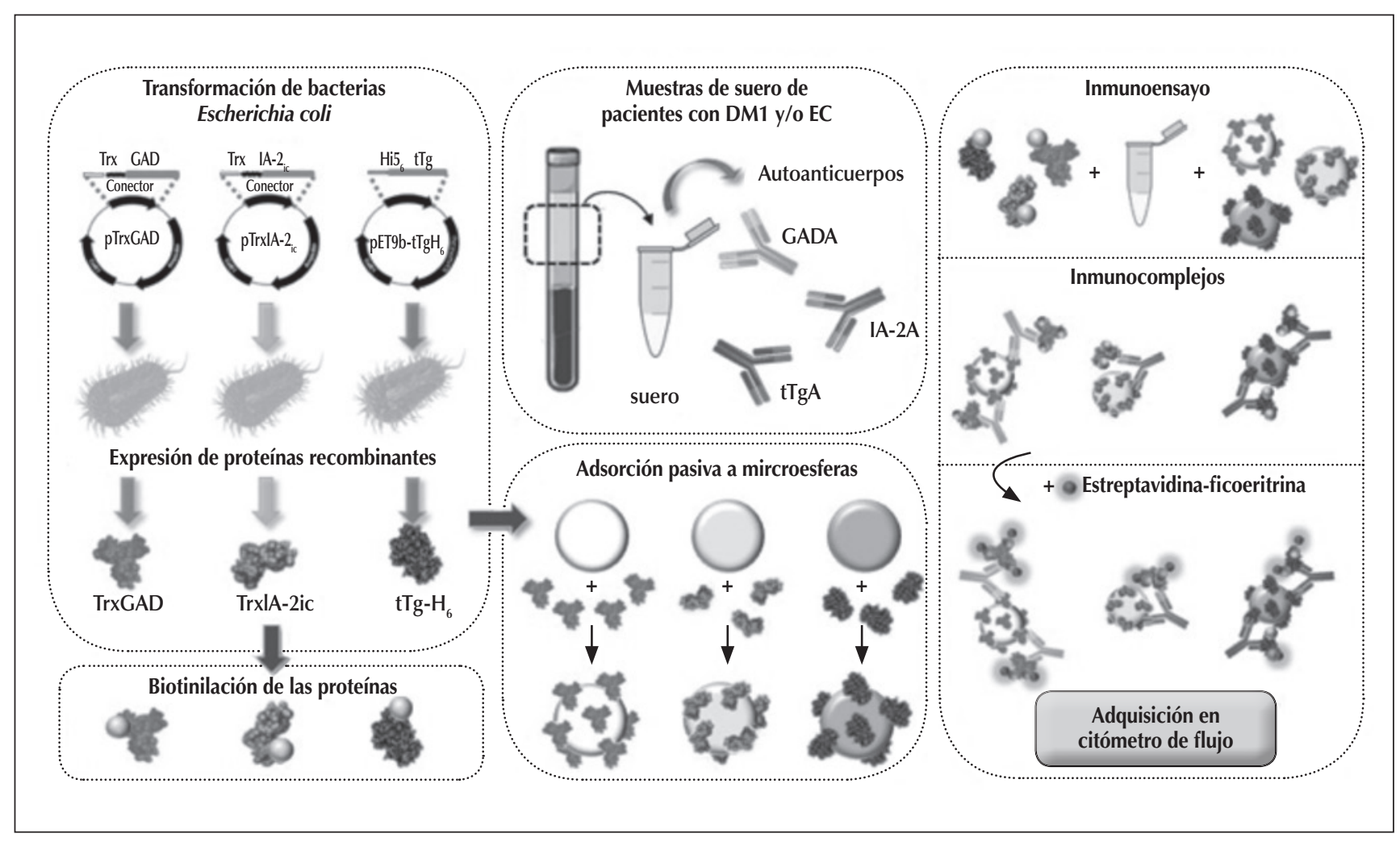

Figura 1: Representación esquemática del diseño experimental del FloCMIA multiplex para GADA, IA-2A y tTgA. 


\section{OBJETIVOS}

El objetivo del presente trabajo fue desarrollar un inmunoensayo basado en citometría de flujo para la detección simultánea (en un único análisis) y discriminativa de dos de los principales marcadores de autoinmunidad involucrados en DMA (GADA e IA-2A) y el más prevalente en EC (tTgA). Las metodologías analíticas basadas en el uso de etiquetas fluorescentes representan herramientas de alta sensibilidad y versatilidad para el estudio de los autoanticuerpos GADA, IA-2A y tTgA. En este sentido, el empleo de microesferas de diferente tamaño y/o fluorescencia interna que pueden discriminarse fácilmente en el citómetro de flujo, adsorbidas con cada uno de los autoantígenos recombinantes, es una herramienta analítica interesante para el estudio simultáneo de estos marcadores en colecciones de sueros de pacientes con DMA/EC.

\section{MATERIALES Y MÉTODOS \\ Serotecas empleadas}

Las muestras de sangre se recolectaron de individuos luego de un ayuno de $8 \mathrm{~h}$ y el suero correspondiente se almacenó a -20。 $\mathrm{C}$ hasta su evaluación. Se emplearon las siguientes serotecas:

- Individuos controles normales provenientes de donantes de sangre sanos sin historia personal o familiar de enfermedades autoinmunes (SHN, $n=35$ ), provistos por el Servicio de Hemoterapia del Hospital de Clínicas "José de San Martín", UBA (Programa con aprobación de los Comités de Investigación y Docencia, y de Ética del mismo Hospital). Dichos sueros se utilizaron para determinar el cut-off en cada uno de los ensayos.

- Pacientes argentinos infanto juveniles con reciente diagnóstico de DM1, diagnosticados según el criterio de la Organización Mundial de la Salud (OMS) $(n=21)^{8}$, provenientes de un programa local establecido con el Servicio de Nutrición del Hospital Nacional de Pediatría R. Gutiérrez (Buenos Aires, Argentina). Dicho programa cuenta con la participación de 15 centros de salud ubicados en la Ciudad Autónoma de Buenos Aires, Gran Buenos Aires y el interior del país. El programa contó con la aprobación del Comité de Ética de dicho hospital. Las muestras ingresaron al Servicio desde mayo de 2013 hasta marzo de 2015, e incluyó un grupo de 14 mujeres y 7 varones. La edad media al diagnóstico fue de $10 \pm 4$ años y el rango etario 1-16 años.
- Pacientes argentinos con reciente diagnóstico de celiaquía. Se emplearon tres sueros de pacientes adultos con reciente diagnóstico de enfermedad celíaca como controles positivos del ensayo.

Para la optimización del Flow Cytometric Microspher-based Immunoassay (FloCMIA multiplex) se seleccionaron sueros con distintas combinaciones de positividad para cada uno de los anticuerpos evaluados (GADA/IA-2A/tTgA: +/+/+; +/+/-; +/-/+; $-/+/+;+/-/-;-/+/-;-/-/+\mathrm{y}-/---)$.

\section{Determinación de marcadores para DMA y EC por los métodos de referencia o consenso internacional}

Los marcadores de DMA, GADA e IA-2A se determinaron empleando los ensayos de unión de radioligando de referencia $(\mathrm{RBA})^{9,10}$.

El marcador para EC tTgA se determinó con un kit de ELISA comercial (ORG540-Orgentec Diagnostika $\mathrm{GmbH}$ ), considerado el método de consenso internacional.

\section{FloCMIA para la detección simultánea de GADA, IA-2A y tTgA o FloCMIA multiplex}

Expresión, purificación y biotinilación de las proteínas recombinantes TrxGAD, TrxIA-2ic y $\mathrm{H}_{6}-t T g$

Las proteínas recombinantes GAD e IA-2ic se expresaron en $E$. coli como proteínas de fusión con tiorredoxina (TrxGAD y TrxIA-2ic, respectivamente) y se purificaron por cromatografía de afinidad siguiendo el protocolo previamente descripto ${ }^{11,12}$. La resina se basó en un soporte de agarosa modificado covalentemente con óxido de fenilarsina que permitió la unión de proteínas que contenían residuos de ditiol vecinos, tal como la $\operatorname{Tr} \mathrm{X}^{13}$. Las preparaciones típicas de TrxGAD y TrxIA-2ic proporcionaron $\approx 10 \mathrm{mg}$ de proteína recombinante por litro de cultivo.

La proteína recombinante $\mathrm{tTg}$ se expresó en $E$. coli fusionada con 6 histidinas $\left(\mathrm{H}_{6}-\mathrm{tTg}\right)$. Luego de la lisis de las bacterias, la fracción intracelular soluble resultante se aplicó a una columna de 1,5 cm x 5,0 cm de Sepharose-FF cargada con iones níquel, previamente equilibrada en buffer $\mathrm{NaH}_{2} \mathrm{PO}_{4} 50 \mathrm{mM}, \mathrm{pH}$ 7,8, $\mathrm{NaCl}$ 0,3 M (buffer 1) con el agregado de imidazol $20 \mathrm{mM}$. Luego de $1 \mathrm{~h}$ de incubación a $4{ }^{\circ} \mathrm{C}$ en agitación, la fracción no retenida se eluyó y la columna se lavó dos veces con $2 \mathrm{ml}$ de buffer 1 con el agregado de imidazol $20 \mathrm{mM}$ y tres veces con $2 \mathrm{ml}$ de buffer 1 con el agregado de imidazol $80 \mathrm{mM}$. Las proteínas unidas fueron eluídas con 5 volúmenes de buffer de elución $\left(\mathrm{NaH}_{2} \mathrm{PO}_{4} 50 \mathrm{mM}, \mathrm{pH} 7,8, \mathrm{NaCl}\right.$ 0,3 M, 
imidazol 200 mM). La preparación típica de $\mathrm{H}_{6}$-tTg proporcionó $\approx 10 \mathrm{mg}$ de proteína recombinante por litro de cultivo.

Dos mililitros de cada proteína recombinante purificada por afinidad se sometieron a intercambio de buffer a buffer fosfato salino isotónico (PBS: $\mathrm{KH}_{2} \mathrm{PO}_{4} 1,5 \mathrm{mM}, \mathrm{Na}_{2} \mathrm{HPO}_{4} 8,1 \mathrm{mM}, \mathrm{NaCl}$ $140 \mathrm{mM}, \mathrm{KCl} 2,7 \mathrm{mM}, \mathrm{pH} 7,4)$ utilizando una columna de desalinización ZEBA (Pierce Biotechnology, Rockford, IL, EE.UU.), de acuerdo con las instrucciones del fabricante. Las proteínas en PBS se incubaron durante $2 \mathrm{~h}$ a $0^{\circ} \mathrm{C}$ con 0,32 mg de sulfoNHS-biotina (Pierce Biotechnology, Rockford, IL, EE.UU.) y se retiró la biotina libre con una nueva columna de desalinización ZEBA.

\section{Protocolo de adsorción pasiva}

Este protocolo se realizó siguiendo las instrucciones del fabricante con modificaciones menores. La adsorción de cada autoantígeno recombinante se efectuó por separado sobre distintas microesferas, cada una conteniendo diferente intensidad de fluorescencia interna teniendo en cuenta las siguientes condiciones: $100 \mu \mathrm{g}$ de una preparación purificada de TrxGAD por cada $100 \mathrm{~cm}^{2}$ de microesferas $(5 \mu \mathrm{L}$ de suspensión de microesferas de $4 \mu \mathrm{m}$ 5\% PN -población 6-, Spherotech, Inc., Lake Forest, IL, EE.UU.), $60 \mu \mathrm{g}$ de una preparación purificada de TrxIA-2ic por cada $100 \mathrm{~cm}^{2}$ de microesferas $(5 \mu \mathrm{L}$ de suspensión de microesferas de $4 \mu \mathrm{m} 5 \%$ PN -población 2-) y $60 \mu \mathrm{g}$ de una preparación purificada de $\mathrm{H}_{6}$-tTg por cada $100 \mathrm{~cm}^{2}$ de microesferas $(5 \mu \mathrm{L}$ de suspensión de microesferas de $4 \mu \mathrm{m}$ 5\% PN -población 4-).

Se emplearon alícuotas de cada una de las proteínas almacenadas con aprotinina 0,1\% PN, en un volumen final de $100 \mu \mathrm{L}$ en buffer isotónico salino (BIS) $\left(\mathrm{NaH}_{2} \mathrm{PO}_{4} 2,3 \mathrm{mM}, \mathrm{Na}_{2} \mathrm{HPO}_{4} 14,2 \mathrm{mM}\right.$, $\mathrm{NaCl} 140 \mathrm{mM}, \mathrm{KCl} 3,8 \mathrm{mM}, \mathrm{pH} 7,4)$. Luego de una incubación durante toda la noche a $4^{\circ} \mathrm{C}$ en agitación, las preparaciones se centrifugaron a $10.000 \mathrm{rpm}$ durante 10 min a $4^{\circ} \mathrm{C}$ y los sobrenadantes se descartaron. Para bloquear los sitios libres sobre la microesfera, los pellets se resuspendieron con $200 \mu \mathrm{L}$ de una solución de seroalbúmina bovina (BSA) 0,5\% $\mathrm{P} N$ en PBS y se incubaron durante $1 \mathrm{~h}$ a temperatura ambiente en agitación. Las preparaciones se centrifugaron de nuevo a $10.000 \mathrm{rpm}$ durante $10 \mathrm{~min}$ a $4^{\circ} \mathrm{C}$; el sobrenadante se desechó y el pellet se lavó con $400 \mu \mathrm{L}$ de PBS. Finalmente se añadieron $200 \mu \mathrm{L}$ de PBS (resultando en $1 \times 10^{5} \mathrm{mi}-$ croesferas -TrxGAD o TrxIA-2ic o H6-tTg / $\mu \mathrm{L}$ ) y las suspensiones se almacenaron a $4^{\circ} \mathrm{C}$ hasta su uso.

Protocolo de FloCMIA multiplex para la detección simultánea y discriminativa de GADA, IA-2A y $t \operatorname{Tg} A$

Excepto cuando se indica de otro modo, todas las etapas de lavado se realizaron con $200 \mu \mathrm{L}$ de PBS-Tween 20 0,05\% VN en el Multiscreen HTS vacuum manifold, y las diluciones de los reactivos se prepararon utilizando BSA 0,5\% P/N, Tween 20 0,05\% VN en PBS y se agregaron $50 \mu \mathrm{L}$ de volumen de reactivos por pocillo en cada incubación. Veinte microlitros de suero humano puro se mezclaron con $15 \mu \mathrm{L}$ de una suspensión que contenía una mezcla de microesferas-TrxGAD $\left(1 \times 10^{5}\right.$ microesferas $\left./ \mu L\right)$, microesferas-TrxIA-2ic $\left(1 \times 10^{5}\right.$ microesferas $\left./ \mu \mathrm{L}\right)$ y microesferas $\mathrm{H}_{6}$-tTg $\left(1 \times 10^{5}\right.$ microesferas $\left./ \mu \mathrm{L}\right)$ y $20 \mu \mathrm{L}$ de una mezcla conteniendo 1,15 ng de TrxGAD-biotina, 2,75 ng de TrxIA-2ic-biotina y 2,50 ng de $\mathrm{H}_{6}$-tTg-biotina. La incubación se realizó durante toda la noche a $4^{\circ} \mathrm{C}$ en agitación. Luego la mezcla reactiva se transfirió a una placa de filtración Multiscreen HTS-HV de 96 pocillos humedecida con PBS. Posteriormente se realizaron cinco lavados con PBS. La detección de los anticuerpos GADA, IA-2A y tTgA unidos requirió de una incubación de $1 \mathrm{~h}$ a temperatura ambiente con 50,00 ng de estreptavidina-ficoeritrina (PE) (Affimetrixe Bioscience, San Diego, CA, EE.UU.) para la detección simultánea de las moléculas de TrxGAD biotina, $\operatorname{Tr} x \mid A-2 i c-b i o t i n a ~ y ~ H_{6}$-tTg-biotina unidas. Después de este paso, la placa se lavó cuatro veces, más una etapa final de lavado con $200 \mu \mathrm{L}$ de PBS y las microesferas se resuspendieron con otros $200 \mu \mathrm{L}$ de PBS. Se realizó la transferencia de la suspensión a tubos de Röhren y se adquirió en un citómetro de flujo PAS III PARTEC. Las muestras se analizaron utilizando el software Cyflogic, se identificó la población de microesferas por gating en un dot plot de forward scatter (FSC) vs side scatter (SSC). Las diferentes poblaciones de las microesferas se discriminaron de acuerdo a la intensidad de la fluorescencia interna de cada una en el canal FL4 (Figura 2), y luego las señales de fluorescencia correspondientes a la emisión de la ficoeritrina -debidas a la formación del inmunocomplejo- se midieron en el canal FL2 y se informaron como media geométrica de la intensidad de fluorescencia (GeoM). 
Los resultados se expresaron como SDs:

$$
S D s=\left(\frac{G e o M_{m}-G e o M_{c}}{D E_{c}}\right)
$$

Donde GeoMm es la GeoM media de las muestras por duplicado, GeoMc es la GeoM media de los sueros de individuos controles normales y DEc su desvío estándar. Se consideraron como muestras GADA, IA-2A o tTgA positivas cuando SDs $>2,5$.

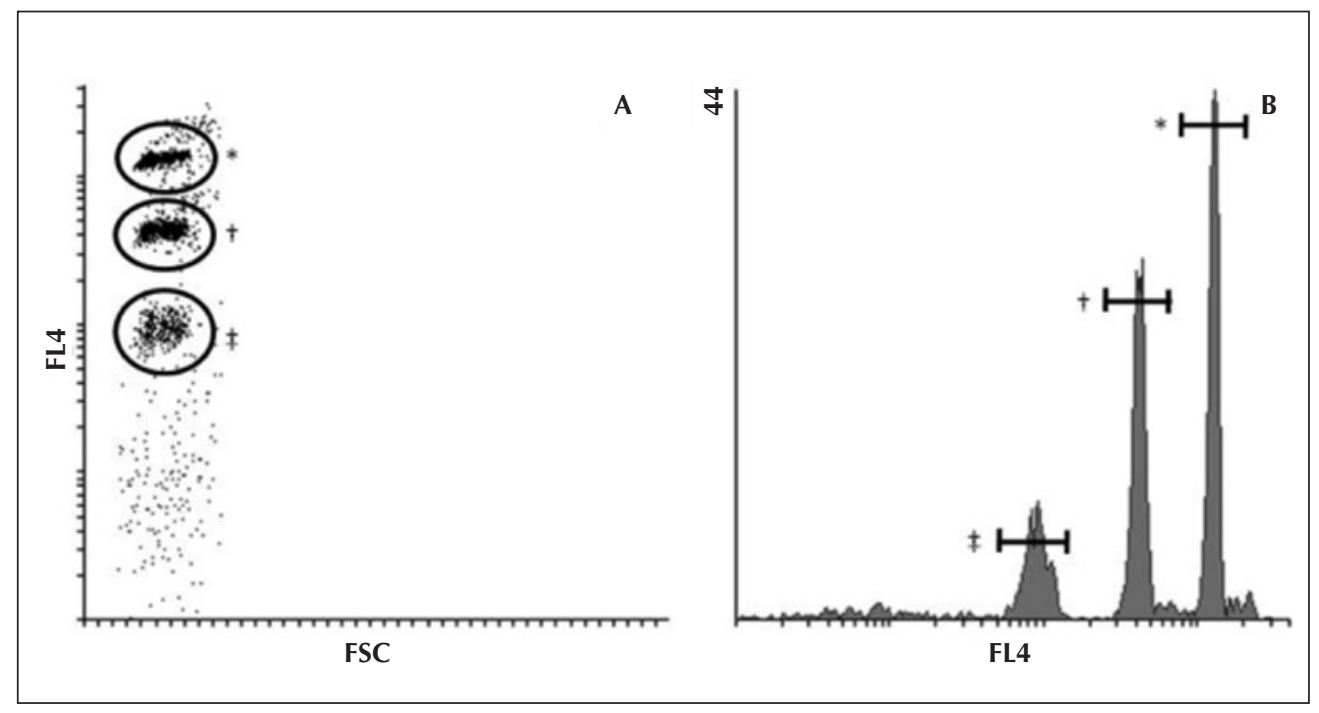

Figura 2: A) Dot plot de intensidad de fluorescencia en el canal FL4 vs forward scatter (FSC). Se realizaron gates sobre cada una de las poblaciones de microesferas (*: población 6 -GAD-; †: población 4 -tTg- y ¥: población 2 -IA-2).

B) Histograma del número de eventos vs intensidad de fluorescencia en el canal FL4 donde puede observarse cada población de microesferas.

\section{Análisis estadístico}

La distribución normal de los datos se analizó mediante el test de normalidad Omnibus de D'Agostino \& Pearson. Se realizó el test de Rout con el fin de eliminar los valores outlier dentro de la población de individuos control normalmente distribuidos. La selección de valores óptimos de cut-off se basó en curvas construidas trazando la especificidad y la sensibilidad calculadas de cada protocolo frente a los valores de corte correspondientes. La significación estadística se evaluó mediante test paramétricos: test $t$ de Student para muestras no pareadas con corrección de Welch. El coeficiente de Spearman $\left(r_{s}\right)$ se calculó para evaluar la correlación entre ensayos. El grado de concordancia entre ensayos se evaluó mediante el cálculo del índice Kappa. Un índice Kappa entre 0,01-0,20 es indicativo de un acuerdo pobre; 0,210,40 acuerdo leve; 0,41-0,60 acuerdo moderado; 0,61-0,80 acuerdo bueno; 0,81-1,00 acuerdo casi perfecto o perfecto ${ }^{14-16}$. Los cálculos se realiza- ron con GraphPad Prism versión 6.01 para Windows (GraphPad Software, San Diego California, EE.UU., www.graphpad.com). Se consideró estadísticamente significativo un valor de $p<0,05$.

\section{RESULTADOS}

\section{Detección de GADA, IA-2A y tTgA por los métodos de referencia $o$ consenso internacional}

Mediante la determinación de GADA por el método de referencia, de los 21 sueros de pacientes con DM1, 17 resultaron positivos para la presencia de dicho marcador $(80,95 \%)$ empleando un cut-off de SDs>3,0 (Figura 3 A). Los niveles de autoanticuerpos se caracterizaron por presentar una SDs mediana $=16,62$ y un rango de señales de 0,43 a 57,67 (Tabla 1). Por otro lado, al analizar la presencia de IA-2A en este mismo grupo, 16 muestras resultaron positivas para dicho marcador $(76,19 \%)$ empleando un cut-off de SDs $>3,0$ (Figura 3 B). Los parámetros observados para 
este ensayo incluyeron una SDs mediana $=12,84$ y un rango de señales de $-1,55$ a 36,47 (Tabla1). Por otra parte empleando el kit comercial para la determinación de tTgA, de los 21 sueros de pacientes con DM1, ocho muestras resultaron positivas para la presencia de dicho marcador $(38,1 \%)$ empleando un cut-off de SDs>3,0 (Figura $3 \mathrm{C}$ ). Los niveles de autoanticuerpos exhibieron una SDs mediana $=16,02$ y un rango de señales de $-0,066$ a 101,7 (Tabla 1). De esta forma se establecieron seis grupos experimentales con diferentes combinaciones de positividad para los distintos autoanticuerpos estudiados (GADA/IA-2A/tTgA): $+/+/+(n=4) ;+/-/-(n=2) ;-/+/-(n=3) ;+/+/-(n=8) ;$ $+/-/+(n=3) ;-/+/+(n=1)$ (Figura 4$)$, los cuales se sometieron posteriormente al análisis por el método FloCMIA multiplex.

El análisis en paralelo de 35 sueros de individuos controles normales confirmó una especificidad del $100 \%$ para GADA e IA-2A, y $97,1 \%$ para tTgA.

Cabe aclarar que las tres muestras utilizadas como controles positivos para la enfermedad celíaca resultaron positivas para tTgA empleando el kit de ELISA comercial.

\section{Detección de GADA, IA-2A y tTgA por FloCMIA multiplex}

Empleando el ensayo FloCMIA multiplex (Figura $5 \mathrm{~A}$ ), de los 21 sueros de pacientes con DM1, 16 resultaron positivos para GADA $(76,2 \%)$ empleando un cut-off de SDs>2,5. Los niveles de autoanticuerpos presentaron una SDs mediana $=14,45$ y un rango de señales de $-1,24$ a 127,92 (Tabla 2). La sensibilidad analítica fue del $88,24 \%$, mientras que el análisis en paralelo de 35 sueros de individuos controles normales determinó una especificidad del 85,71\%, con la eliminación de cinco muestras con señales outlier para el cálculo del cut-off. Por otro lado, al analizar la presencia de IA-2A en este mismo grupo (Figura 5 B), 11 muestras resultaron positivas para dicho marcador $(52,38 \%)$ empleando un cut-off de SDs $>2,5$. Los parámetros observados para este ensayo incluyeron una SDs mediana $=3,64$, un rango de señales de $-0,94$ a 36,85 y una sensibilidad analítica del 56,25\% (Tabla 2). El análisis en paralelo de 35 sueros de individuos controles normales confirmó una especificidad del $85,71 \%$ con la eliminación de cinco muestras con señales outlier para el cálculo del cut-off.

En relación al marcador de EC tTgA, el ensayo de FloCMIA multiplex mostró que de los 21 pacientes con DM1, nueve resultaron positivos para la presencia de dicho marcador $(42,86 \%)$ empleando un cut-off de SDs $>2,5$. Los niveles de autoanticuerpos exhibieron una SDs mediana $=1,53$ y un rango de señales de -2,29 a 107,55 (Tabla 2). La sensibilidad analítica fue del $50 \%$, mientras que el análisis en paralelo de 35 sueros de individuos controles normales determinó una especificidad del $80,0 \%$ con la eliminación de siete muestras con señales outlier para el cálculo del cut-off. De los tres sueros de pacientes con enfermedad celíaca utilizados como controles positivos, dos resultaron positivos empleando el método de FloCMIA multiplex.

\section{Resultados integrados y análisis de correlación}

El diagrama de Venn (Figura 6 A) ilustra los resultados integrados para la determinación de GADA por RBA y FloCMIA multiplex. Del análisis de las 21 muestras de pacientes con DM1, 15 fueron positivas por ambos métodos $(71,43 \%)$, mientras que tres resultaron negativas por ambos ensayos (14,29\%). El RBA fue capaz de detectar como positivas dos muestras que el diseño multiplex de FloCMIA no pudo establecer como positivas, sin embargo se destaca que el método FloCMIA fue capaz de determinar la presencia del marcador en un suero que el RBA no pudo. Luego del análisis de los resultados integrados de la determinación de IA-2A por RBA y FloCMIA multiplex en las 21 muestras de pacientes con DM1, se observó que nueve sueros fueron positivos por los dos métodos $(42,9 \%)$, mientras que otros tres resultaron negativos por ambos (14,29\%) (Figura 6 B). El RBA fue capaz de detectar como positivas a siete muestras que el diseño multiplex de FloCMIA no pudo establecer como positivas. Cabe destacar que el método de FloCMIA detectó dos muestras como positivas que el RBA no logró visualizar como positivas. A pesar de las diferencias en los principios fisicoquímicos de las técnicas RBA y FloCMIA utilizadas para la determinación de GADA e IA-2A, se observó alguna correlación entre los ensayos para cada uno de los marcadores; coeficiente de Spearman: $r_{s}$ GADA $=0,4859$ $(p<0,0001)$ y $r_{s} \mid A-2 A=0,3233(p<0,0151)$. Asimismo el valor del índice Kappa fue de 0,683 para GADA y 0,387 para IA-2A, representando un grado de concordancia bueno y leve entre los métodos 


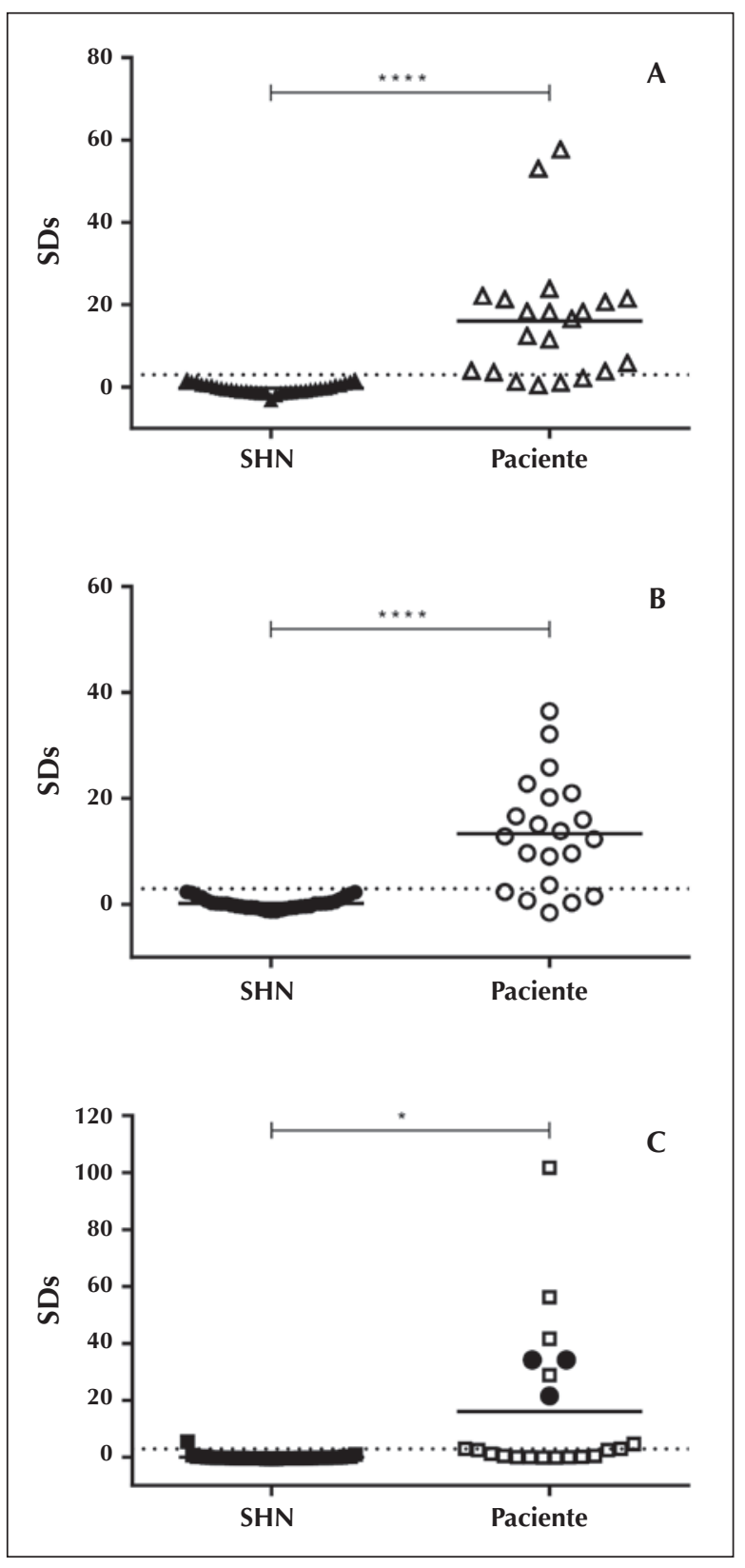

**** $p<0,0001$ y ${ }^{*} p<0,05$ estadísticamente significativo.

Figura 3: Distribución de las señales obtenidas por RBA para GADA (A) e IA-2A (B) y un kit de ELISA comercial para $\operatorname{tg} \mathrm{A}(\mathrm{C})$, realizados en 21 sueros de pacientes con DM1 y en $35 \mathrm{SHN}$. Los resultados se expresaron como score de desvío estándar (SDs). Los tres círculos negros (๑) que se observan corresponden a pacientes deficientes en $\lg \mathrm{A}$, SDs informado corresponde a anticuerpos del isotipo lgG anti-tTg. En todos los gráficos la línea punteada indica el cut-off del ensayo (cut-off $=3,0$ ) y la línea continua las medianas para cada población. respectivamente. Es así que la correlación entre métodos para determinar GADA e IA-2A es real y no por muestreo azaroso.

Por otra parte, para el marcador tTgA tanto el kit de ELISA comercial como el FloCMIA multiplex lograron detectar como positivos a cuatro pacientes con reciente diagnóstico de DM1 (19\%). Sin embargo hubo ocho sueros (38\%) que fueron negativos para ambos (Figura 6 C). Es interesante destacar que, a pesar que el ELISA logró evidenciar como positivas cuatro muestras que FloCMIA multiplex no pudo, este nuevo inmunoensayo logró detectar la presencia del marcador de EC en cinco muestras (24\%) informadas como negativas por el método de consenso internacional. De esta manera el valor del índice Kappa fue de 0,211 , el cual es indicativo de un grado de concordancia leve entre ensayos.

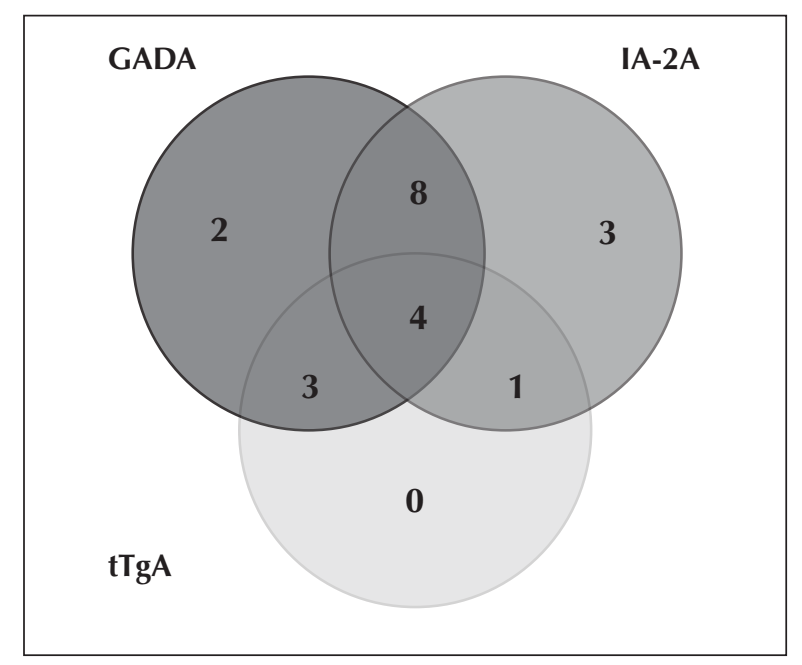

Figura 4: Diagrama de Venn de los resultados obtenidos para la determinación de GADA e IA-2A por RBA y tTgA por ELISA sobre una población de 21 pacientes infanto juveniles con reciente diagnóstico de DM1. En este diagrama se establecen seis grupos experimentales según la presencia (+) o ausencia (-) del marcador correspondiente. Los sueros negativos para los tres marcadores corresponden a los $35 \mathrm{SHN}$ ensayados. 


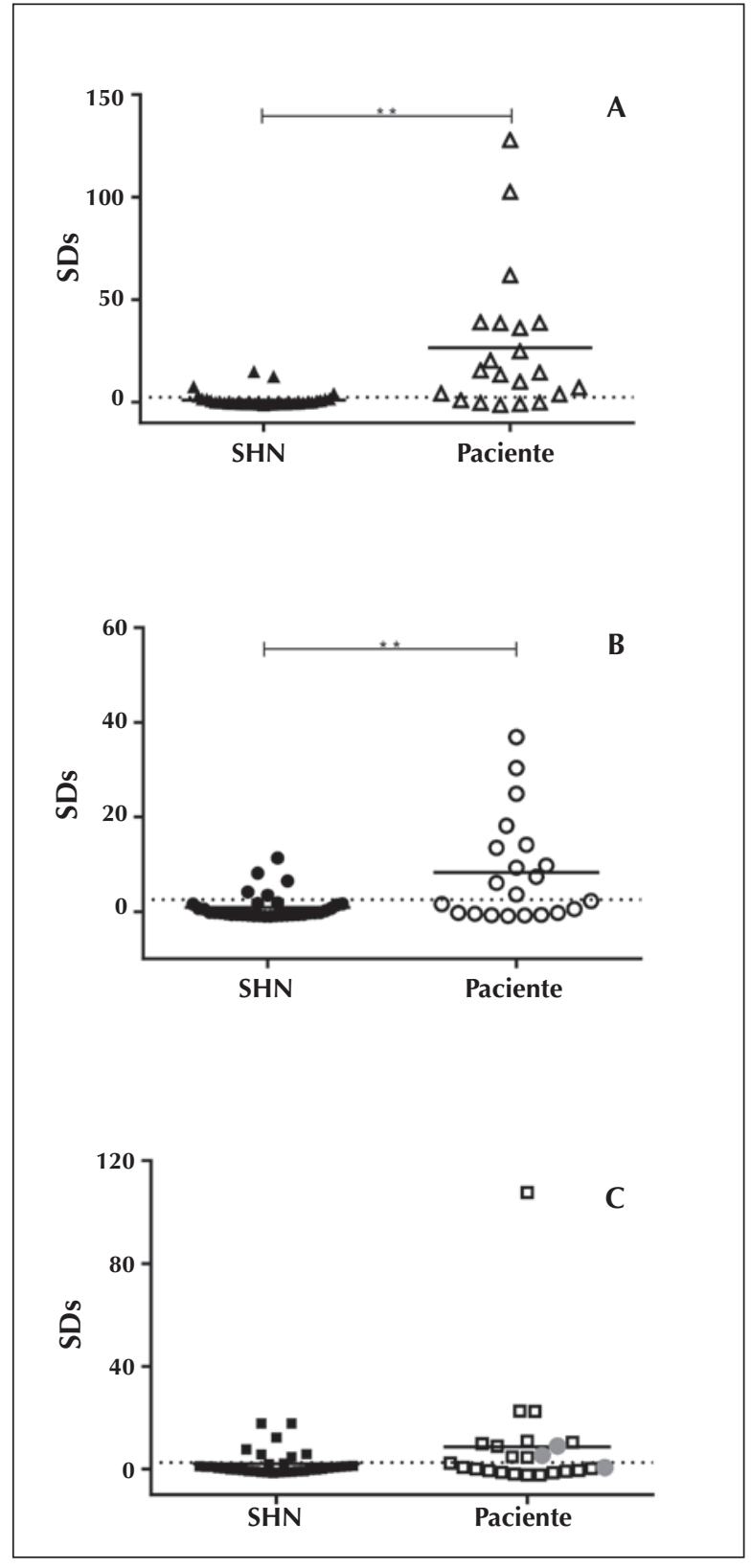

${ }^{*} p<0,01$ estadísticamente significativo.

Figura 5: Distribución de las señales obtenidas empleando el FloCMIA multiplex para GADA (A), IA-2A (B) y $\operatorname{tTg} A(C)$, realizado en 21 sueros de pacientes con DM1 y en 35 SHN. Los resultados se expresaron como score de desvío estándar (SDs). En todos los casos la línea punteada indica el cut-off del ensayo (cut-off $=2,5$ ). En el gráfico $C$ se observan tres círculos grises (๑) que corresponden a los controles positivos del ensayo.

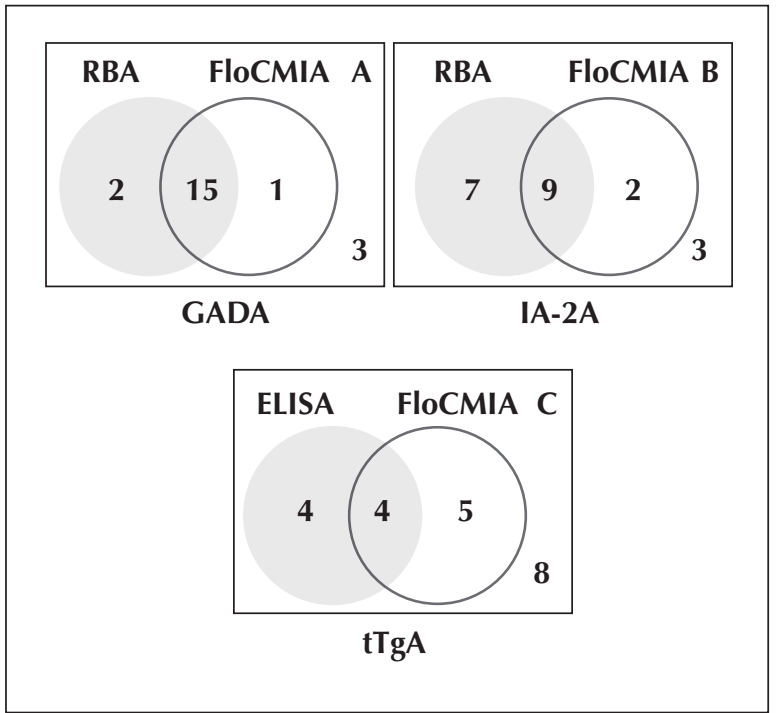

Figura 6: Diagramas de Venn de los resultados integrados para la determinación de GADA (A), IA-2A (B) y tTgA $(C)$ realizados por RBA (GADA e IA-2A) o por ELISA (tTg) y FloCMIA multiplex empleando 21 sueros de pacientes infanto juveniles con reciente diagnóstico de DM1.

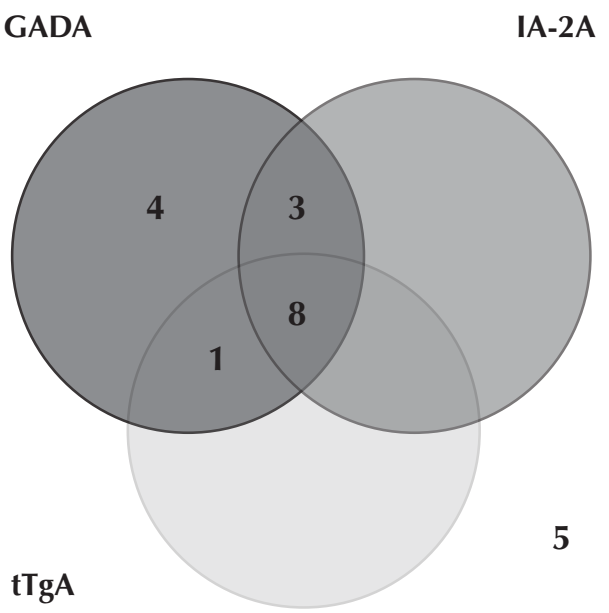

Figura 7: Diagrama de Venn de los resultados integrados para la determinación de GADA, IA-2A y/o tTgA en 21 sueros de pacientes infanto juveniles con reciente diagnóstico de DM1, realizado por el método FloCMIA multiplex. 


\begin{tabular}{|l|l|l|l|}
\hline \multicolumn{1}{|c|}{ Inmunoensayo } & \multicolumn{1}{c|}{ RBA } & \multicolumn{1}{c|}{ ELISA } \\
\hline \multicolumn{1}{|c|}{ Autoanticuerpo } & \multicolumn{1}{|c|}{ GADA } & \multicolumn{1}{c|}{ ITgA } \\
\hline Mediana (SDs) & 16,62 & 12,84 & 16,02 \\
\hline Rango (SDs) & $0,43-57,67$ & $-1,55-36,47$ & $-0,07-101,70$ \\
\hline $\begin{array}{l}\% \text { de pacientes DM1 } \\
\text { detectados como positivos }{ }^{\mathrm{a}}\end{array}$ & 80,95 & 76,19 & 38,00 \\
\hline Especificidad (\%) $^{\mathrm{b}}$ & 100 & 100 & 97,10 \\
\hline
\end{tabular}

a Porcentaje de pacientes diabéticos detectados como positivos para GADA e IA-2A por RBA, y para tTgA por el kit de ELISA (n=21).

${ }^{b}$ La especificidad se calculó como 100 menos el porcentaje de sueros humanos normales detectados como positivos $(n=35)$.

Tabla 1: Parámetros analíticos obtenidos para GADA e IA-2A por RBA y para tTgA por ELISA comercial.

\begin{tabular}{|l|l|l|l|}
\hline \multicolumn{1}{|c|}{ Autoanticuerpo } & \multicolumn{1}{c|}{ GloCMIA multiplex IA-2A } & \multicolumn{1}{c|}{ tTgA } \\
\hline Mediana (SDs) & \multicolumn{1}{|c|}{ GADA } & \multicolumn{1}{c|}{ IA } & 1,53 \\
\hline Rango (SDs) & 14,45 & $-0,64-36,85$ & $-2,29-107,55$ \\
\hline $\begin{array}{l}\text { \% de pacientes DM1 } \\
\text { detectados como positivos }\end{array}$ & $-1,24-127,92$ & 52,38 & 42,86 \\
\hline Sensibilidad analítica $(\%)^{\mathrm{b}}$ & 76,19 & 56,25 & 50,00 \\
\hline Especificidad (\%) & 88,24 & 85,71 & 80,00 \\
\hline
\end{tabular}

a Porcentaje de pacientes diabéticos detectados como positivos para cada marcador utilizando el método de FloCMIA multiplex.

${ }^{b}$ Porcentaje de pacientes positivos por RBA (GADA e IA-2A) o por ELISA (tTgA) que fueron detectados como positivos utilizando el método de FloCMIA multiplex.

Tabla 2: Parámetros analíticos obtenidos para GADA, IA-2A y tTgA por el método FloCMIA multiplex.

\section{DISCUSIÓN}

El novedoso FloCMIA multiplex para la detección de autoanticuerpos reportado en esta comunicación representa un gran progreso hacia la simplificación para el screening de la población a gran escala para la detección de DMA y EC. El ensayo multiplex basado en citometría de flujo tiene una serie de características atractivas comparado con la combinación de los actuales métodos individuales de referencia o consenso internacional que incluyen: 1) la capacidad para detectar dos enfermedades: DMA y EC; 2) la reducción de los costos y el tiempo operativo respecto de la realización de las tres determinaciones individuales al ofrecer una alternativa más económica y socialmente inclusiva a las poblaciones de bajos recursos; 3) la no utilización de precursores radiactivos; 4) la habilidad para detectar autoanticuerpos de todas las clases y subclases de inmunoglobulinas; 5) la posibilidad de incorporar este método en laboratorios de mediana/ baja complejidad que ya posean citómetro de flujo.

El método de referencia para la detección de los marcadores de DMA es el ensayo de unión de radioligando (RBA), el cual presenta alta sen- sibilidad y especificidad ${ }^{17}$. Sin embargo el uso de material radiactivo -altamente contaminante para el ambiente, no biodegradable y de elevado costohace que sea difícil implementarlo en laboratorios de mediana/baja complejidad, por lo cual estos estudios se limitan a laboratorios de referencia y la comunidad médica depende de la derivación de muestras a dichos laboratorios.

Por su parte los ELISAs comerciales para la determinación de tTgA no permiten la detección de todos los isotipos de lg, empleándose la detección del isotipo IgA anti-tTg, el cual aparece aumentado en la mayoría de los casos. El FloCMIA multiplex desarrollado se basa en el diseño conocido como "doble paratope" con el cual se logra la detección de autoanticuerpos de todas las clases y subclases de inmunoglobulinas. Esto es posible dado que, en este tipo de ensayos, el anticuerpo se une por uno de sus paratopes al antígeno recombinante inmovilizado en las microesferas y por el otro paratope al antígeno soluble biotinilado ${ }^{18,19}$. Este aspecto es sumamente importante en el diagnóstico de EC, dado que un porcentaje de los pacientes es deficiente en el isotipo IgA de 
anticuerpos, los cuales son los que se detectan con los kits comerciales actualmente disponibles en el mercado. El protocolo actual para el diagnóstico de enfermedad celíaca indica que se debe cuantificar IgA total junto con la determinación de tTgA (lo cual implica más tiempo, reactivos y dinero) dado que un resultado negativo para estos autoanticuerpos puede deberse a una deficiencia de IgA. Así, utilizando el ensayo aquí presentado no es necesario realizar el dosaje de IgA porque el FloCMIA multiplex detecta tanto lgA como lgG.

Otra ventaja del método desarrollado es la capacidad de detectar en forma simultánea y discriminativa, en un único acto analítico, los principales marcadores de autoinmunidad de DM y/o EC logrando así una estrategia de mayor sensibilidad diagnóstica. Esto tiene un impacto directo para el médico solicitante, no sólo porque puede conocer el perfil de autoinmunidad de su paciente con una sola determinación analítica, sino también porque puede solicitar el análisis de la combinatoria de marcadores que crea convenientes de acuerdo a la clínica y edad de cada paciente. Por lo tanto, este desarrollo tiene como principal beneficiario al paciente quien, de acuerdo a los resultados obtenidos, podrá recibir el tratamiento adecuado a su patología en forma oportuna, mejorar sustancialmente su calidad de vida y reducir así la aparición de las complicaciones a largo plazo.

Las condiciones del FloCMIA multiplex empleadas en el presente estudio se basaron en protocolos previos de ensayos FloCMIA singleplex desarrollado por nuestro laboratorio para el marcador GADA ${ }^{19}$. Empleando microesferas de diferente fluorescencia interna adsorbidas con cada uno de los antíge-

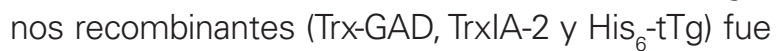
posible acomodar tres ensayos diferentes de autoanticuerpos en una sola determinación con una pequeña cantidad de muestra de suero $(20 \mu \mathrm{l})$. La sensibilidad (76,19\% para GADA, 52,38\% para IA$2 A$ y $42,86 \%$ para $t$ TgA) y la especificidad $(85,71 \%$ para GADA, $85,71 \%$ para IA-2A y $80,00 \%$ para tTgA) del FloCMIA multiplex fueron comparables a las obtenidas con cada uno de los ensayos de referencia o consenso. En cuanto a los rangos dinámicos obtenidos para cada marcador con el FloCMIA multiplex (GADA: -1,24-127,92; IA-2A: -0,94-36,85 y tTgA: $-2,29-107,55)$ fueron en general más amplios que los obtenidos con los RBA y kit comercial.

Actualmente no existe en el mercado un kit diagnóstico que tenga la capacidad de determinar en un único acto analítico y en forma discriminati- va los marcadores de autoinmunidad de estas dos enfermedades genéticamente relacionadas (DMA y EC). El inmunoensayo FloCMIA multiplex sería el primer desarrollo en nuestro país de un kit para el diagnóstico y seguimiento de estas dos patologías relacionadas. Esto representa una gran ventaja operativa ya que el $50 \%$ de los laboratorios de hospitales públicos de CABA posee citómetro de flujo, por lo cual podrán incluir estudios de marcadores de autoinmunidad en muestras de pacientes para caracterizarlos en forma rápida y reducir los costos y tiempos operativos de las tres determinaciones individuales. El desarrollo de FloCMIA multiplex, en el formato de kit de diagnóstico, posibilitará la sustitución de kits importados (costo actual: U\$S 200-2.000 para 50 determinaciones) abaratando sensiblemente los costos de los análisis.

Otro aspecto novedoso es su versatilidad que posibilita incorporar la determinación de otros anticuerpos presentes en EC (anti-péptidos deamidados, anti-gliadina) o en otras enfermedades autoinmunes relacionadas, que pueden darse en simultaneidad en estos pacientes como la enfermedad tiroidea autoinmune (anti-tiroglobulina (Tg), peroxidasa tiroidea (TPO) y el receptor de la tirotropina (TSHR).

\section{CONCLUSIONES}

Hemos desarrollado un ensayo multiplex para medir con precisión tres autoanticuerpos en una sola determinación usando un pequeño volumen de sangre, lo que facilitará el screening de la población general a gran escala en forma simultánea para DMA y EC. Las condiciones del FloCMIA multiplex presentado deberán optimizarse aún más y será necesaria una validación a gran escala antes que este ensayo pueda aplicarse para la detección en forma masiva.

La importancia crucial de este desarrollo radica en el mejoramiento de la performance analítica para la detección de marcadores de autoinmunidad, con una considerable repercusión en términos de apoyo diagnóstico a la bioquímica asistencial endocrinológica y en el impacto socioeconómico.

\section{Agradecimientos}

Agradecemos a las Dras. Liliana Trifone y Adriana Roussos del Servicio de Nutrición del Hospital de Pediatría Ricardo Gutiérrez y a la División de Hemoterapia del Hospital de Clínicas José de San Martín (Buenos Aires, Argentina) por recolectar y suministrar los sueros de pacientes diabéticos y celíacos y de individuos controles normales respectivamente. 


\section{Financiamiento}

Este trabajo fue financiado en parte por los siguientes subsidios: Subsidio Especial para Investigación sobre Diabetes y Celiaquía de la Sociedad Argentina de Diabetes, año 2016; UBACYT 2016 Mod I, 20020150100115BA; Proyectos de Investigación Científica y Tecnológica de la Agencia Nacional de Promoción Científica y la Tecnológica (PICT-2014-1928).

\section{BIBLIOGRAFÍA}

1. Palmer JP, Hirsch IB. What's in a name: Latent autoimmune diabetes of adults, type 1.5, adult-onset, and type 1 diabetes. Diabetes Care 2003; 26:536-538. doi:10.2337/diacare.26.2.536.

2. Zimmet PZ, Shaten BJ, Kuller LH, et al. Antibodies to glutamic acid decarboxylase and diabetes mellitus in the multiple risk factor intervention trial. American Journal of Epidemiology 1994; 140:683-690. doi:10.1093/oxfordjournals.aje.a117316.

3. Lohi S, Mustalahti K, Kaukinen K, et al. Increasing prevalence of coeliac disease over time. Alimentary Pharmacology \& Therapeutics 2007; 26:1217-1225. doi:10.1111/j.13652036.2007.03502.x

4. Fasano A, Berti I, Gerarduzzi T, et al. Prevalence of celiac disease in at-risk and not-at-risk groups in the United States: a large multicenter study. PubMed - NCBI. Arch Intern Med 2003; 163:286-292. Disponible en: https://www.ncbi.nlm.nih.gov/ pubmed/12578508.

5. Husby S, Koletzko S, Korponay-Szabó IR, et al. European Society for Pediatric Gastroenterology, Hepatology, and Nutrition Guidelines for the Diagnosis of Coeliac Disease. Journal of Pediatric Gastroenterology and Nutrition 2012; 54:136-160. doi:10.1097/MPG.0b013e31821a23d0.

6. Bao F, Yu L, Babu S, et al. One third of HLA DQ2 homozygous patients with type 1 diabetes express celiac disease-associated transglutaminase autoantibodies. Journal of Autoimmunity 1999; 13:143-8. doi:10.1006/jaut.1999.0303.

7. Chiang JL, Kirkman MS, Laffel LMB, et al. Type 1 diabetes through the life span: a position statement of the American Diabetes Association. Diabetes Care 2014; 37:2034-2054. doi:10.2337/dc14-1140.

8. Diabetes mellitus. Report of a WHO Study Group. World Health Organization Technical Report Series 1985; 727:1-113. Disponible en: http://www.ncbi.nlm.nih.gov/pubmed/3934850.

9. Grubin CE, Daniels T, Toivola B, et al. A novel radioligand binding assay to determine diagnostic accuracy of isoform-specific glutamic acid decarboxylase antibodies in childhood IDDM. Diabetologia 1994; 37:344-50. Disponible en: http://www.ncbi. nlm.nih.gov/pubmed/8063033.
10. Gianani R, Rabin DU, Verge CF, et al. ICA512 Autoantibody radioassay. Diabetes 1995; 44:1340-1344. doi:10.2337/ diab.44.11.1340.

11. Papouchado ML, Valdez SN, Ghiringhelli D, et al. Expression of properly folded human glutamate decarboxylase 65 as a fusion protein in Escherichia coli. European Journal of Biochemistry 1997; 246:350-9. Disponible en: doi:10.1111/j.14321033.1997.00350.x.

12. Guerra LL, Faccinetti NI, Trabucchi A, et al. Novel prokaryotic expression of thioredoxin-fused insulinoma associated protein tyrosine phosphatase 2 (IA-2), its characterization and immunodiagnostic application. BMC Biotechnology 2016; 16:84. Disponible en: doi:10.1186/s12896-016-0309-2.

13. Hoffman RD, Lane MD. lodophenylarsine oxide and arsenical affinity chromatography: new probes for dithiol proteins. Application to tubulins and to components of the insulin receptor-glucose transporter signal transduction pathway. The Journal of Biological Chemistry 1992; 267:14005-11.

14. Cohen J. Weighted kappa: nominal scale agreement with provision for scaled disagreement or partial credit. Psychological Bulletin 1968; 70:213-20. Disponible en: http://www.ncbi.nlm. nih.gov/pubmed/19673146.

15. Landis JR, Koch GG. The measurement of observer agreement for categorical data. Biometrics 1977; 33:159-74. Disponible en: http://www.ncbi.nlm.nih.gov/pubmed/843571.

16. Koch GG, Landis JR, Freeman JL, et al. A general methodology for the analysis of experiments with repeated measurement of categorical data. Biometrics 1977; 33:133-58. Disponible en: http://www.ncbi.nlm.nih.gov/pubmed/843570.

17. Törn C, Mueller PW, Schlosser M, et al. Diabetes Antibody Standardization Program: evaluation of assays for autoantibodies to glutamic acid decarboxylase and islet antigen-2. Diabetologia 2008; 51:846-52. doi:10.1007/s00125-008-0967-2.

18. Villalba A, Valdez SN, lacono RF, et al. Development of 2 alternative enzyme-linked immunosorbent assays for routine screening of glutamic acid decarboxylase autoantibodies. Clinica Chimica Acta; International Journal of Clinical Chemistry 2007; 376:82-7. doi:10.1016/j.cca.2006.07.017.

19. Guerra LL, Faccinetti NI, Bombicino SS, et al. Novedoso inmunoensayo multiplex por citometría de flujo para la detección simultánea y discriminativa de GADA e IA2A en pacientes con Diabetes Mellitus. Revista de la Asociación Bioquímica Argentina, Bioquímica y Patología Clínica 2018; 82:14-26. Disponible en: https://www.aba-online.org.ar/ejemplares-revistabypc-2018/revista-aba-vol-82-n-1-ene-abril-2018. 